ORIGINAL ARTICLE

\title{
Stress fractures of the femoral shaft in women's college lacrosse: a report of seven cases and a review of the literature
}

\author{
L Kang, D Belcher, M J Hulstyn
}

Br J Sports Med 2005;39:902-906. doi: 10.1136/bjsm.2004.016626

See end of article for authors' affiliations

Correspondence to: Dr Hulstyn, Brown University Department of Orthopaedics Division of Sports Medicine, 2 Dudley St, Providence, RI 02905, USA; hulstyn@cox.net

Accepted 19 April 2005
Background: Stress fractures do not often occur in the shaft of the femur. They are more common in the femoral neck, the tibial shaft, the metatarsals, and other bones of the foot. In female athletes, stress fractures classically afflict the distance runner, the ballerina, the gymnast, and the figure skater.

Objectives: To describe the clinical presentation, diagnosis, treatment, and outcome of seven college female lacrosse players with femoral shaft stress fractures, and review the literature.

Results: The unusual results of this study support the principle that clinical suspicion should be high when treating any female athlete regardless of the sport. In this case series, an abrupt change in the quality of the running surface during the competitive training season was the only underlying common thread among the athletes.

Conclusion: The findings suggest that risk factors for the female athlete are variable and are no longer limited to the undernourished or overtrained.
S tress fractures are common in today's active and athletic population. Originally identified in military recruits, stress fractures are now recognised as overuse injuries of the athlete. ${ }^{2}$ In most cases, they involve the tibia and metatarsals, but an estimated $7-10 \%$ occurs in the femur. Most stress fractures of the femur afflict distance runners in whom they occur in the femoral neck, whereas those that occur in the shaft are relatively uncommon. ${ }^{45}$ However, a few studies have suggested that stress fractures of the femoral shaft occur more often than is generally appreciated. ${ }^{67}$

The female athlete constitutes a subgroup of athletes with an increased susceptibility to stress fractures. ${ }^{8} 9$ The potential risks for this are numerous and include overtraining, an imbalance of hormones, and a chronic low oestrogen state that results in decreased bone density. The "female triad" syndrome is a specific risk factor for stress fractures and is characterised by the combination of disordered eating, amenorrhoea, and osteoporosis. ${ }^{8}{ }^{9}$ Historically, the typical female athlete who is at risk of developing a stress fracture has been a ballet dancer, a gymnast, a figure skater, or a marathon runner. ${ }^{10-15}$ These sports are typically involved because of the emphasis on weight restrictions, body image, and/or aesthetics. Participants thus perceive weight gain to have a negative impact on performance, and weight loss is believed to be a positive risk factor for stress fractures. The increase in women's participation in recreational and competitive athletics, however, may change the pattern, frequency, and distribution of stress fractures so that their occurrence will increase in other individual and team sports. The lower number of stress fractures reported in female team based sports, such as lacrosse and soccer, implies that the risk of stress fractures is not as high as in individual sports. Low suspicion, under-reporting, and the conception that team based sports place less emphasis on body image are likely causes for this lack of data. ${ }^{816}$

Previous reports of femoral shaft fractures have involved either military recruits during basic training ${ }^{17-19}$ or long distance runners. ${ }^{30-14}$ There is one case report of bilateral distal femur fractures in a 15 year old male rugby player. ${ }^{20}$ We present here a unique occurrence of stress fractures of the femoral shaft in seven collegiate female lacrosse players, six of which occurred within the span of three consecutive seasons.

\section{MATERIALS AND METHODS}

Seven cases were retrospectively reviewed. Patient data were collected from medical charts and radiographic files. Information on the training schedule, characteristics of the running surface, and quality of shoe wear was obtained from the coaches and trainers.

Athletes were characterised as overtrainers, undertrainers, or cross trainers. Overtrainers doubled the winter workout assignment and ran three to five miles in addition to the official workout directed by the team coach. Athletes were undertrainers if they performed less than or the basic minimum of what was assigned. Cross trainers were particularly dedicated to alternating the types of exercise performed and the surfaces on which they ran.

Routine physical examination was performed on all the athletes. In addition, the fulcrum test was performed when a femoral shaft fracture was suspected. In this test, the patient's femur is levered by the examiner on the examiner's arm. A positive finding is the elicitation of pain by this manoeuvre and is highly sensitive for stress factors of the femoral shaft. ${ }^{7}$

Weight, height, and body mass index (BMI) were determined. BMI measures the weight/height ratio in kilograms divided by the square of the height in metres. Ideal weight is defined as a BMI of 18.7-24.9 for all adults regardless of age. A BMI below 18.7 is classified as underweight. ${ }^{21}$ BMI values of 25-30 are classified as overweight, and above 30 is classified as obese.

Plain radiographs of the involved femur were taken in all cases. A bone scan and/or magnetic resonance imaging (MRI) scan were obtained to aid in the diagnosis. Serum laboratory studies were performed in only one case to determine whether a metabolic abnormality had resulted from rapid

Abbreviations: $\mathrm{BMI}$, body mass index; $\mathrm{MRI}$, magnetic resonance imaging 
Table 1 Characteristics and diagnostic studies for each female lacrosse player studied

\begin{tabular}{|c|c|c|c|c|c|c|c|}
\hline & Case 1 & Case 2 & Case 3 & Case 4 & Case 5 & Case 6 & Case 7 \\
\hline Class year & 2nd & 2nd & 2nd & Freshman & 2nd & 2nd & Freshman \\
\hline Height ( $\mathrm{ft} /$ in) & 55 & 59 & 511 & 54 & 52 & 510 & 56 \\
\hline Weight (lb) & 120 & 126 & 155 & 135 & 120 & 160 & 133 \\
\hline $\mathrm{BMl}^{*}$ & 20.0 & 18.6 & 21.6 & 23.2 & 21.9 & 23.0 & 21.6 \\
\hline Side & Right & Left & Right & Left & Right & Left & Right \\
\hline Position & Midfield & Attack & Attack & Defence & Midfield & Midfield & Attack \\
\hline Training style & Undertrainer & Overtrainer & Undertrainer & Overtrainer & Cross trainer & Cross trainer & $\begin{array}{l}\text { Undertrainer then an } \\
\text { overtrainer* }\end{array}$ \\
\hline Eating disorder & No & Yes & No & No & No & No & \\
\hline Menstrual cycle & Normal & Abnormal & Abnormal & Normal & Normal & Normal & Normal \\
\hline Fulcrum test & Not performed & + & + & + & + & + & + \\
\hline Radiograph & - & + & - & - & - & - & + \\
\hline Bone scan & + & + & & & + & + & \\
\hline MRI & & + & + & + & + & & \\
\hline
\end{tabular}

weight loss in conjunction with active disordered eating (anorexia nervosa).

An internet search using three different weather history data sources ${ }^{22-24}$ was performed to determine the average city temperatures during the initial months of the official lacrosse practice season. This was correlated with the frozen conditions of the playing field as reported by the coaches and trainers.

\section{RESULTS}

All seven athletes were white members of the varsity women's lacrosse team with an age range of 19-23. Height and weight varied considerably, but six of the seven BMI values were normal. Only one patient admitted to both an eating disorder (anorexia nervosa) and abnormal menses. Another patient reported exercised induced oligomenorrhoea without an associated eating abnormality. One patient was taking oral contraceptives electively. The fulcrum test was positive in six cases; in the single case where a femoral shaft fracture was not suspected, the test was not performed (table 1).

All stress fractures occurred at the same institution under the supervision of the same group of team coaches and trainers. Six of the seven fractures occurred between April

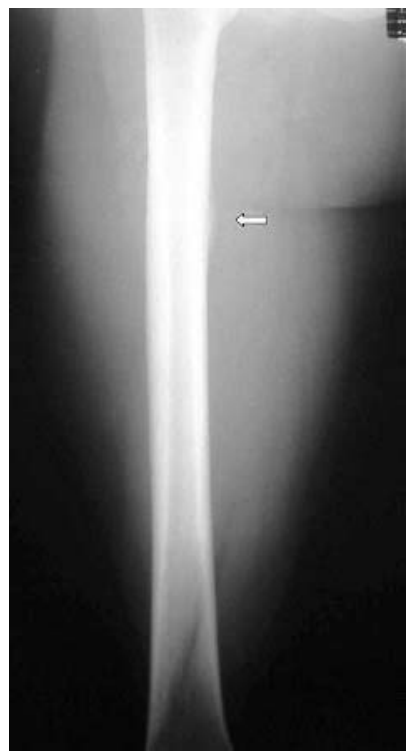

Figure 1 Anterior-posterior radiograph of the right femur showing periosteal reaction at the medial proximal third femoral shaft.
2001 and April 2003; one case occurred in 1998. All fractures presented and were diagnosed either in the middle or the end of the competitive season between March and early May. Confirmation of the diagnosis was provided by plain radiographs in two cases (fig 1), by bone scan in four cases (fig 2), and by MRI (fig 3) in five cases (table 1).

The yearly training schedule was as follows. Between 15 September and 15 November during the collegiate academic year, the team coach held 12 official practices which consisted of drills, conditioning, weight lifting, and two scrimmage games. On the off days-that is, when practices were not run by the team coach-practices were led by the team captains and consisted of either a three to four mile run or an intrateam scrimmage.

The winter training schedule from 15 November to 31 January consisted of daily assigned workout routines which included the following: (1) a warm up before the workout; (2) aerobic interval conditioning with prescribed time intervals, target heart rates, and weekly frequencies by means of (a) sprinting, running, and jogging, $(b)$ swimming, (c) cycling, (d) a stair-master, or (e) a treadmill; (3) weight training exercises; (4) stretching after the workout. Because

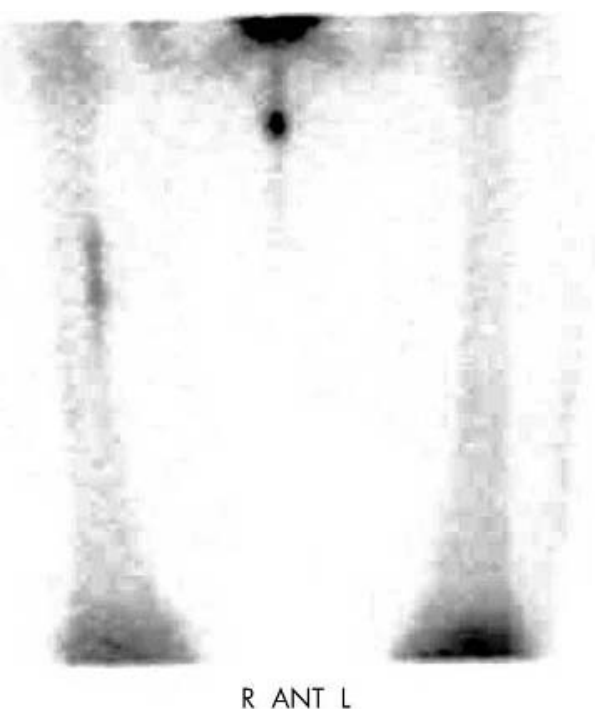

Figure 2 Bone scan showing increased activity of the right medial proximal third femoral shaft. 
these practices were unsupervised and based on guidelines only, there was considerable variability among the team members in the amount, quality, and intensity expended during the workout. Three athletes were overtrainers, two were undertrainers, and two were cross trainers (table 1).

The official competitive season began on 1 February and ended in mid to late April. During this period, official practices with the team coaches lasted about two hours a day and included a total of three to five miles of running a day. The total number of official practices led by the team coaches was limited to 20 days during the competitive season. The remainder of the scheduled practices consisted of unofficial practices led by the team captains.

During the first six weeks of the official season (from February to mid-March), athletes changed practice surfaces from an indoor track to the outdoor frozen field, and the exact timing of this transition depended on permissible weather conditions. The indoor track was composed of rubber cement. The outdoor track on the roof of the gymnasium was an artificial rubber turf that the athletes used when the fields were still not usable. The outdoor frozen field was typically ready for use during the month of March.

The results of the weather history data web search were as follows. The average city temperature during the month of February is $29.7^{\circ} \mathrm{F}$ with the average number of days below freezing being $24 .^{25}{ }^{26}$ The average high temperature during February is $38^{\circ} \mathrm{F}$, and the average low is $22^{\circ} \mathrm{F} \cdot{ }^{27}$ In contrast, the average city temperature during the month of March is $37.4^{\circ} \mathrm{F}$, with the average number of days during this month below freezing being $19 .^{25}{ }^{26}$ The average high temperature in March is $46^{\circ} \mathrm{F}$, and the average low is $29^{\circ} \mathrm{F} .{ }^{27}$

All team members wore high quality footwear. This included only top of the line running shoes for both indoor and outdoor long distance runs, turf shoes for the rubber artificial turf, and moulded cleats for outdoor field play.

\section{DISCUSSION}

Since the 1970s, the nature of stress fractures has changed from being considered to be a "military disease" to being more commonly observed in athletes of all ages, sex, and sports. This change has paralleled the trend of modern day society towards a more active and athletic lifestyle and applies to both women and men. It also reflects the increased awareness among clinicians and the advances in imaging technology to detect these fractures. With the rise of female participation in sports, women are just as likely to suffer from stress fractures as men. ${ }^{8}$

The case series presented here is unique for several reasons. First is that all cases involved participants of the same athletic team within a relatively brief span of time. Six of the seven cases occurred within three consecutive seasons at a rate of two cases a year. The training environment for these women was short and intense and placed them at greater risk of fracture. Provost and Morris ${ }^{4}$ defined the "fatigue" (or stress) fracture as one that "begins as a gradual dissolution of bone as a result of repeated sub-maximum and generally unaccustomed stresses which may or may not result in a complete fracture". At a histological level, it has been suggested that stress fractures are focal structural weaknesses occurring during bone remodelling in response to the repeated application of subthreshold stresses where the rate of osteoclastic resorption is greater than the rate of new bone formation. ${ }^{28}$ Boden and Speer $^{2}$ provide a modern day description of stress fractures as the end result of an imbalance between bone injury and bone remodelling. This more general definition perhaps reflects the fact that the exact mechanism responsible for initiating stress fractures remains unclear. One leading theory proposes that excessive forces are transmitted to the bone when the surrounding muscles become fatigued. ${ }^{23}$ This relatively weak muscle is believed to reduce the shock absorbing capacity of the lower extremity and to permit the redistribution of forces to bone. An alternative theory states that highly concentrated muscle forces act across a localised area of bone, causing mechanical insults above the stress bearing capacity of the bone. These insults occur at the insertion of tendons such as the adductor magnus and the medial head of the gastrocnemius where excavations in bone propagate into stress factors. ${ }^{23}$

It is likely that in this group of patients, all of the above mechanisms played a contributory role. This multifactorial aspect of stress fractures is paralleled by the fact that there was considerable variability in the intensity and frequency of preseason and in-season training among the seven athletes. Potentially, those classified as undertrainers were at increased risk of fracture because of the greater transfer of forces to the bone by the surrounding weak muscle. This contrasts with the situation of the three overtrainers whose increased risk was theoretically the result of an inordinate amount of repetitive forces placed on their bones in excess of their capacity. These generalisations do not take into

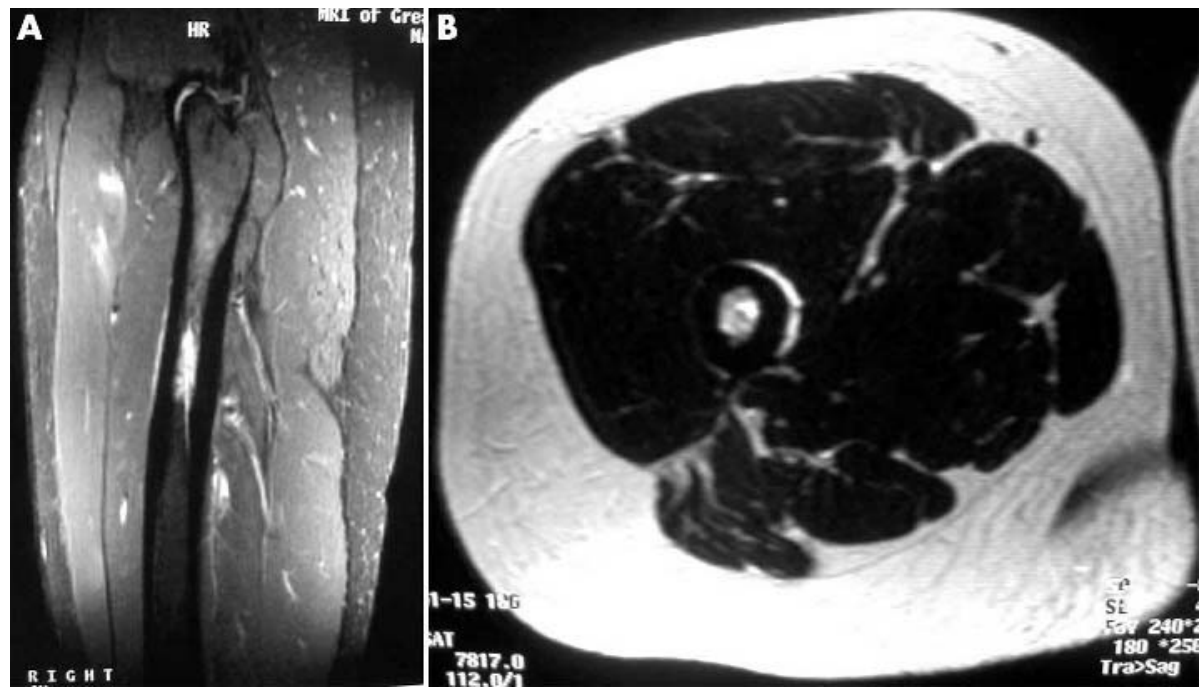

Figure 3 (A) A coronal magnetic resonance imaging (MRI) scan showing increased signalling of the marrow. (B) An axial MRI showing marrow oedema and inflammation in the surrounding periosteum. 
consideration differences in running biomechanics, body weight and mass, bone physiology, nutritional status, and running surface,[25-27 29-31] which also appear to play a role (see below). Other potential risk factors that this study was not designed to assess are the anatomical features of the foot and ankle, such as forefoot varus, leg length inequality, and height of the longitudinal arch of the foot, which Korpelainen $e^{2} a^{32}$ have shown increases the risk of recurrent and multiple stress factors in athletes. The relative contribution of these different factors for each individual case remains unclear and needs further investigation.

The second unique feature of this series was that all cases presented shortly after the athletes had switched running surfaces from an indoor track to a frozen outdoor field from February to mid-March. Of all of the risk factors among the cases, this was the only one common to all. This commonality introduces the concept that, in addition to the classic aetiological mechanisms of an imbalance and excess of forces as described above, any abrupt change may serve as an additional mechanism by which stress factors arise. It follows that athletes should be cognisant of sudden or rapid changes in their normal regimen, whether it is in their training schedule, body mass or weight, running surfaces, or the like. Coaches, trainers, and athletes need also to prepare for the transition from the aerobic demands of distance running to the more anaerobic requirements of explosive footwork, such as sprinting and backpedalling, which involve a frequent change in direction and acceleration and deceleration. The specific characteristics that were variable among the cases, such as overtraining (case 2 , case 4 , case 7 ), poor nutrition and altered eating habits (case 2), and undertraining (case 1, case 3, case 7) therefore probably served as individual risk factors which were compounded by the abrupt alteration in running surfaces to which they were exposed.

The third distinguishing feature of this series was that the stress fractures occurred in the shaft and not in the neck of the femur. In the study of Matheson et $\mathrm{al}^{3}$ of 320 athletes, the femur was the fourth most common site of bone involved (7.2\% $v 49.1 \%$ in the tibia), but this number reflected a combination of both the neck and the shaft and failed to differentiate the proportions between the two. This study found a significant difference in the ages of athletes who suffered femoral fractures and those who suffered tibial fractures, with the former occurring in an older population. In contrast, all cases in our series involved young patients aged 19-23. Matheson et $a l^{3}$ also reported no statistically significant association between weekly running mileage and the location of the fracture, although fractures of the femur were associated with the greatest number of miles run. In addition, femur fractures corresponded to a shorter time to both diagnosis and recovery, a fact ascribed by the authors to higher clinical suspicion. Our study suggests that there may be a differential effect between individual and team sports on the location of stress fractures of the femur analogous to the differential effect of individual and team sports on athletic competitive anxiety. ${ }^{16}$ As today's society produces a greater number of participants in all areas of athletic activity, it will be interesting to determine whether the demographics of these participants directly correlate with a particular pattern of fracture location.

The physical findings of localised tenderness and swelling are notoriously absent from femoral shaft fractures. We found this to be the case in this study. This is also consistent with the report of Matheson et al, ${ }^{3}$ which showed no statistically significant association between diagnosis and local tenderness or swelling. The authors attributed this to the bulk of overlying soft tissues. Moreover, the fulcrum test proved to be much more critical in making the diagnosis, which is a finding that is consistent with previous studies.?

\section{What is already known on this topic}

- Stress fractures of the femoral shaft are less common than in the tibia, metatarsals, and femoral neck and are usually treated non-operatively

- Diagnosis and recognition are crucial in the management of these fractures

\section{What this study adds}

- This study introduces the concept that a sudden and abrupt change in an athlete's training regimen is an important factor in the development of stress fractures

- It also supports the notion that female athletes may have not only a higher risk of stress fractures, in general, but a higher risk of stress fractures localised to the femoral shaft as well

When performed, this test was positive and led to further imaging studies with radiographs, bone scan, or MRI. Not surprisingly, plain radiographs were not always sensitive. Rather, this study confirmed the greater utility and accuracy of both the bone scan and the MRI in the work up of suspected femur fractures. ${ }^{351213}$

This unique set of cases described in our study bears clinical significance by illustrating what happens when there exists a mismatch between what is prescribed and what is actually performed. This mismatch resulted in the athletes being either undertrained or overtrained and has prompted the coaches, trainers, and team doctors to explore ways to minimise it. The discrepancy between what is spoken and what is perceived lends even more support to the need to optimise both in season and off season physical and mental preparation and to effect open communication among all involved participants. Despite the change in the nature of the stress fracture over the decades from one that was almost exclusive to military recruits, to one more common among long distance runners, to one that no longer discriminates among athletes, what has remained constant over time is that early detection is most often the key to successful nonoperative treatment. Fortunately, all of the cases presented here were diagnosed before fracture displacement, and all responded to conservative measures. Further education and more lucid communication may be crucial to prevention, which may be the only way to reduce the incidence of stress fractures in our increasingly active society.

Finally, it is clinically relevant that the female triad was not the most common thread in our series and that all three components of the triad were not fully present among this small group of women. Although case 2 intimated the essence of the female triad syndrome, only two of the three disorders of the female triad (weight loss and disordered eating), by definition, were present, as osteoporosis was ruled out by a normal bone density study. Yet, this patient still went on to sustain a stress fracture. An important conclusion from our study is that poor nutrition, altered hormones, abnormal menstrual cycles, and disordered eating are potential problems in any female sport and vary in the degree of severity. These conditions are often undiagnosed, ${ }^{8} 9$ and perhaps the original concept of the female triad should now be viewed as embodying a spectrum of potential risk factors among today's female athletic population. The typical 
athlete at risk of stress fractures is no longer limited to a gymnast, ballet dancer, or figure skater. Women are now joining all ranks of sports, and this has resulted in a concomitant increase in media exposure. Admittedly, the degree of this public exposure pales in comparison with the publicity received by men's sports, but the psychological pressure among female athletes to maintain a certain body image in the public eye is perhaps greater than that for male athletes. Although the "psycho-physiology" that underlies the female triad is not the focus of this study, it should be stressed that it continues to be a problem of which coaches, trainers, and team doctors should show heightened awareness and deliver appropriate treatment. They should also now be looking at uncommon locations and in all types of athlete when stress fractures are suspected.

\section{Authors' affiliations}

L Kang, Hospital for Special Surgery, New York, USA

D Belcher, Department of Athletics, Brown University, Providence, RI, USA

M J Hulstyn, Department of Orthopaedics, Brown University

Competing interests: none declared

\section{REFERENCES}

1 Brethaupt ZVR. Pathologie monschuchew fusser. MED Zeittung 1855:24:169-75.

2 Boden BP, Speer KP. Femoral Stress Fractures. Clin Sports Med 1997; 16:307-17

3 Matheson GO, Clement DB, McKenzie DC, et al. Stress fractures in athletes: a study of 320 cases. Am J Sports Med, 1987; 15:46-58.

4 Provost RA, Morris JM. Fatigue fracture of the femoral shaft. J Bone Joint Surg [Am] 1969;51:487-98.

5 Stanitski CL, McMaster JH, Scranton PE. On the nature of stress fracture. Am J Sports Med 1978;6:391-6.

6 Clement DB, Ammann W, Taunton JE, et al. Exercise-induced stress injuries to the femur. Int J Sports Med 1993;14:347-52.

7 Johnson AW, Weiss CB, Wheeler DL. Stress fractures of the femoral shaft in athletes: more common than expected. A new clinical test. Am J Sports Med 1994;22:248-56.

8 Teitz CC, Hu SS, Arendt EA. The female athlete: evaluation and treatment of sports-related problems. J Am Acad Orthop Surg 1997;5:87-96.

9 Voss LA, Fadale PD, Hulstyn MJ. Exercise-induced loss of bone density of athletes. J Am Acad Orthop Surg 1998;6:349-57.
10 Blatz DJ. Bilateral femoral and tibial shaft stress fractures in a runner. Am J Sports Med 1981;9:322-5.

11 Butler JE, Brown SL, McConnell BG. Subtrochanteric stress fractures in runners. Am J Sports Medicine 1982;10:228-32.

12 Lombardo SJ, Benson DW. Stress fractures of the femur in runners. Am J Sports Med 1982;10:219-27.

13 Luchini MA, Sarokhan AJ, Micheli $\sqcup$. Acute displaced femoral shaft fractures in long-distance runners. J Bone Joint Surg [Am] 1983:65:689-91.

14 McBryde AM. Stress fractures in runners. Clin Sports Med 1985:4:737-52.

15 Slemenda CW, Johnston CC. High intensity activities in young women: site specific bone mass effects among female figure skaters. Bone Miner 1993;20:125-32

16 Wong EH, Lox CL. Relation between sports context, competitive trait anxiety, perceived ability, and self-presentation confidence. Percept Motor Skills 1993;76:847-50

17 Bargren JH, Tilson DH, Bridgeford OE. Prevention of displaced fatigue fractures of the femur. J Bone Joint Surg [Am] 1971;53:1115-17.

18 Giladi $M$, Ahronson Z, Stein M, et al. Unusual distribution and onset of stress fractures in soldiers. Clin Orthop 1985;192:142-6.

19 Hallel T, Amit S, Segal D. Fatigue fractures of tibial and femoral shaft in soldiers. Clin Orthop 1976;118:35-43.

20 Yasuda T, Miyazaki K, Tada K, et al. Stress fracture of the right distal femur following bilateral fractures of the proximal fibulas. Am J Sports Med 1992;20:771-4.

21 Heiat A. National Institutes of Health (NIH: the NIH Consensus Conference on Health Implications of Obesity in 1985); United States Department of Agriculture (the 1990 Department of Agriculture' Dietary Guidelines for Americans); National Heart, Lung, and Blood Institute. Prev Cardiol 2003;6:104-7.

22 http://www.cityrating.com (accessed 9 Sep 2005)

23 http://www.usatoday.com/weather/climate/usa/ri/wprovide.htm (accessed 9 Sep 2005).

24 http://www.srh.noaa.gov/tulsa/climate/min32.html (accessed 9 Sep 2005).

25 Krivickas LS. Anatomical factors associated with overuse sports injuries. Sports Med 1997;24:132-46.

26 Orava S, Puranen J, Ala-ketola L. Stress fractures caused by physical exercise. Acta Orthop Scand 1978:49:19-27.

27 Orchard J. Is there a relationship between ground and climatic conditions and injuries in football? Sports Med 2002;32:419-32.

28 Li G, Zhang S, Chen G, et al. Radiographic and histologic analyses of stress fracture in rabbit tibias. Am J Sports Med 1985;13:285-94.

29 Dixon SJ, Batt ME, Collop AC. Artificial playing surfaces research: a review of medical engineering and biomechanical aspects. Int J Sports Med 1999:20:209-18.

30 Ekstrand J, Nigg BM. Surface-related injuries in soccer. Sports Med 1989;8:56-62.

31 Heidt RS, Dormer SG, Cayley PW, et al. Differences in friction and torsional resistance in athletic shoe-turf surface interfaces. Am J Sports Med 1996;24:834-42

32 Korpelainen R, Orava S, Karpakka J, et al. Risk factors for recurrent stress fractures in athletes. Am J Sports Med 2001;29:304-10. 